Forum 2016 $\cdot 31: 326$

DOI 10.1007/s12312-016-0113-x

Online publiziert: 18. Juli 2016

๑) Springer-Verlag Berlin Heidelberg 2016
Interdisziplinäre Arbeitsgemeinschaft für Weichgewebesarkome (IAWS)

\section{Neuer Vorstand gewählt}

\section{Bericht über die Mitgliederversammlung der IAWS}

Die interdisziplinäre Arbeitsgemeinschaft für Weichgewebesarkome (IAWS) in der Deutschen Krebsgesellschaft besteht seit 2006. Im Rahmen der Mitgliederversammlung während des DKK wurde die neue Satzung der Arbeitsgemeinschaft bestätigt und in Kraft gesetzt. Dem bisherigen Vorstand der IAWS wurde einstimmig für seine Arbeit gedankt, und er konnte nach Entlastung verabschiedet werden.

Anschließend wählte die Mitgliederversammlung einen neuen Vorstand, dem als Sprecher Prof. P. Hohenberger aus Mannheim und als Sekretär Prof. H. R. Dürr, LMU München vorstehen. Als Vorstandsmitglieder wurden Prof. P. Reichardt, Sarkomzentrum Berlin-Brandenburg, Prof. B. Kasper, Interdisziplinäres Sarkomzentrum Mannheim, Prof. W. Budach, Klinik für Strahlentherapie Universität Düsseldorf, Herr Dr. B. Jobke, Radiologie am DKFZ (Deutsches Krebsforschungszentrum Heidelberg), Prof. E. Wardelmann, Pathologisches Institut der Universität Münster und Prof. M. Lehnhardt, Berufsgenossenschaftliche Kliniken Bergmannsheil Bochum gewählt.

Dem Vorstand werden außerdem bis zu 7 kooptierte Mitglieder angehören, so z.B. Prof. Sebastian Bauer, Westdeutsches Tumorzentrum Essen als Sprecher der AIO Sarkomgruppe, PD Dr. M. Schuler, Dresden als Vertreter des Bereiches Versorgungsforschung, Vertreter der GPOH/Berlin, sowie PD Dr. U. Dierksen, Münster, als noch zu benennende Mitglieder der Sarkomarbeitsgruppen in der Schweiz und Österreich.

\section{Nationale und internationale Aufgaben}

Vor der neuen Führung der IAWS liegen erhebliche Aufgaben, sowohl versorgungs- als auch strukturpolitischer Art. So werden europaweit Referenznetzwerke für seltene Tumorerkrankungen gefördert und gefordert.
Die European Reference Network (ERNs) wurden gerade gestartet und beinhalten mit EURACAN ein Netzwerk für selten solide Tumoren. Zusätzlich bietet die EU Joint Action on Rare Cancer (JARC) die Möglichkeit Leitlinien, Patientenvertretung, und Außenwirkung von Netzwerken für seltene Tumorerkrankungen zu gestalten. Im Rahmen dieser Maßnahme ist die Deutsche Krebsgesellschaft in Kooperation mit der German Interdisciplinary Sarcoma Group (GISG) und der IAWS gefordert, die existierenden Leitlinien zusammenzutragen, zu bewerten, und ggf. Bedarf für neue Leitlinien zu benennen. Bereits existente und wissenschaftliche valide Leitlinien sollen europaweit allen Ländern in ähnlicher Form zugänglich gemacht werden.

National besteht die Aufgabe, eine S3-Leitlinie für die Therapie von Weichgewebesarkomen zu erstellen. Dies ist federführend zwischen DKG, der AIO-Sarkomarbeitsgruppe und GISG bewilligt und wird durch die Deutsche Krebshilfe gefördert. Das Zweitmeinungsnetzwerk der DKG für Hodentumoren kann als ein Vorbild für die Etablierung qualifizierter Therapien auch für Weichgewebesarkome verstanden werden. Allerdings liegen die Problemstellungen für Sarkome anders als für Hodentumoren. Bildgebung, Tumorlokalisation, histologische Subtypisierung nehmen vor Behandlungsbeginn bei Weichgewebesarkomen einen deutlich anderen Stellenwert ein als für Hodentumoren.

\section{Wer ist die IAWS?}

Die IAWS versteht sich als eine Interdisziplinäre Arbeitsgemeinschaft, in der medizinische Onkologen, Radio-Onkologen, Chirurgische Onkologen, Orthopädische Onkologen, Pathologen, Epidemiologen aber auch Radiologen, Vertreter von Patientenselbsthilfegruppen (z. B. Das Lebenshaus oder SOS-Demoid) und psychoonko- logisch Tätige im Bereich der Therapie von Sarkomen aufgefordert sind sich einzubringen. Auch die Förderung der nicht-ärztlichen Berufe (z. B. Physiotherapeuten, Sarkom-Schwester, Training von study nurses zum Patientenumgang) sind wichtige Aufgaben. Der Schwerpunkt liegt auf den Sarkomen des Erwachsenenalters. Adoleszenten und Jungerwachsenen (AYA, Adolescent and Young Adult), die einer besonders intensiven Betreuung bedürfen, sollen überschneidend mit der GPOH behandelt werden. Der Bereich der pädiatrischen Weichgewebe und vorwiegend Knochentumoren ist in der GPOH sehr gut repräsentiert und abgedeckt.

\section{Rückblick}

Die IAWS hat neben der Planung von Sitzungen für den DKK seit 2010 ein Register für adjuvante und neoadjuvante Therapien unter der Führung von Prof. J.T. Hartmann, Tübingen/Kiel/Bielefeld betrieben. Eine Analyse dieser Register steht aus, um ggf. Daten für die Durchführung prospektiver Studien zu generieren. Die Durchführung von Therapiestudien ist schwerpunktmäßig Aufgabe der GISG (www.gisg. de). Zum Aufgabenbereich gehört auch die Deutschen Sarkomkonferenz, die jährlich im März in Berlin stattfindet. Relevant ist vor allem auch die Beteiligung der Patientenselbsthilfegruppen, des Forschungsnetzwerkes TranSarNet und der Arbeitsgemeinschaft Orthopädische Onkologie und der Arbeitsgemeinschaft Knochentumoren.

\section{Kontakt}

Univ.-Prof. Dr. Peter Hohenberger Leiter der Sektion Spezielle Chirurgische Onkologie \& Thoraxchirurgie

Universitätsmedizin Mannheim

E-mail: peter.hohenberger@umm.de 